\title{
Synchronization properties of Voltage Source Converters when seen as Coupled Oscillators based on the Kuramoto Model
}

\author{
Noe Barrera Gallegos* Victoria Gasca Segura** \\ Marta Molinas *** \\ * Department of Engineering Cybernetics, Norwegian University of \\ Science and Technology, 7491 Trondheim, Norway (e-mail: \\ noebg20@gmail.com). \\ ** Faculty of Electrical Engineering, Electronics and Computer \\ Systems, Universidad Tecnolgica De Pereira, Pereira, Colombia, \\ (e-mail: mvgasca@utp.edu.co) \\ *** Department of Engineering Cybernetics, Norwegian University of \\ Science and Technology, 7491 Trondheim, Norway \\ (marta.molinas@ntnu.no)
}

\section{INTRODUCTION}

Microgrids are present in electrical networks and are becoming more important in the conventional AC distribution networks. Microgrids are composed by converters which transfer the power produced in the microgrids to the loads. It is crucial to control properly the converters in order to ensure stability of the voltage magnitude and frequency on the grid.

The general scheme of the voltage source converter (VSC) is presented in Fig. 1. Using modulated signals to control the pulses for opening and closing the switching devices, the converter is able to transfer back and forth the energy form the DC side to AC grid.

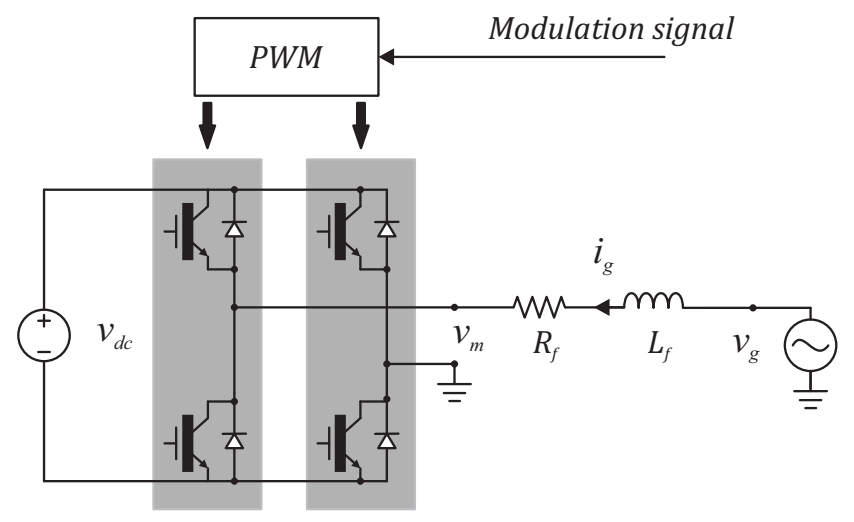

Fig. 1. Voltage source converter

The aim of this paper is to compare the properties of synchronization, here we present the comparison between two control strategies with very different conceptions. One is the synchronous reference frame control (SRFC), which is set by assuming that the converter exhibits a linear behavior [Yazdani and Iravani (2010) Teodorescu et al. (2011)]. The second controller is the virtual oscillator control (VOC). This is a new control approach for the AC/DC converters developed by Johnson et al. (2012). This control strategy uses a nonlinear oscillator that provides a modulation signal for the converter. This comparison is meaningful because both approaches have the same purpose in a microgrid: the control of the power in the converter.

\section{SYNCHRONOUS REFERENCE FRAME CONTROL}

Born for the control of electrical machinery (Kundur (1994)), the rotational framework $d q 0$ helps to transform the sinusoidal values into constant values. This transformation simplify the control of the AC current in the inverters. This transformation is commonly used in three phase circuits. For the single-phase converter an additional step is needed for the phase angle detection. A fictitious $\alpha-\beta$ signal is produced by using the second order generalized integrator (SOGI). The resultant signals in quadrature provide a proper way to transform the single phase voltage and current into the $d q 0$ framework.

\subsection{Droop control}

Under the assumption that the current control is tuned, and the PLL has a faster time response than the current control, then the power angle of the converter $\delta_{i}$ is small (De Brabandere et al. (2004)). It is also assumed that the resistance in the output filter $R_{s}$ is negligible. Therefore, the power expression for the converter is,

$$
S_{i}=P_{i}+j Q_{i}
$$

Assuming that $\delta_{i}$ is small, the active power $P_{i}$ is approximated by,

$$
P_{i} \approx \frac{V_{g} V_{m} \delta_{i}}{X_{L}}
$$

and the approximated reactive power $Q_{i}$ is,

$$
Q_{i} \approx\left(\frac{V_{g}-V_{m}}{X_{L}}\right) V_{g}
$$


These relationships allow the proportional control for the voltage and frequency in the converter terminals by applying the following proportional control laws.

$$
\begin{gathered}
V_{m}=V_{m}^{*}+m_{Q i} Q_{i} \\
\omega=\omega^{*}+m_{P i} P_{i}
\end{gathered}
$$

Where $m_{Q i}$ and $m_{P i}$ are the proportional droop gains for voltage and frequency respectively.

\subsection{Kuramoto equivalent model}

The equivalent Kuramoto model is the representation of the oscillatory characteristics of coupled oscillators (also called Kuramoto oscillators). Consider the phase of the $i^{t h}$ converter connected in parallel to the utility grid, which follows the dynamic.

$$
\frac{d \theta_{i}}{d t}=\omega_{i}-\sum_{j=1}^{n} a_{i j} \sin \left(\theta_{i}-\theta_{j}\right)
$$

Each converter connected to the utility grid has a degree of coupling $a_{i j}$ to the utility grid frequency. The coupling parameter $a_{i j}$ for each converter is by definition (SimpsonPorco et al. (2012)),

$$
a_{i 0} \triangleq \frac{V_{g} V_{m}}{X_{L}}
$$

Where $V_{g}$ and $V_{m}$ are the RMS values of the voltages in the utility grid and the converter $\mathrm{AC}$ side.

\section{VIRTUAL OSCILLATOR CONTROL}

The virtual oscillator control works using a resonant circuit with equations similar to the nonlinear van Der pol oscillator.

The dynamic is that of a parallel RLC circuit and a nonlinear voltage dependent current source (Johnson et al. (2016)). Where the resonant circuit and the dependent current source $g\left(v_{c}\right)$ are implemented in a programmable device (computer, FPGA, etc.), the program provides the modulation signals to the converter. The current and voltage equations are,

$$
\begin{gathered}
L \frac{d i_{L}}{d t}=v_{c} \\
C \frac{d v_{c}}{d t}=\sigma v_{c}-k_{l} v_{c}^{3}-\frac{v_{c}}{R}-i_{L}+k_{l} u(t)
\end{gathered}
$$

Where the term $\left(\sigma v_{c}-k v_{c}^{3}\right)$ describe the nonlinear dynamic of the voltage dependent current source, $v_{c}$ is the voltage in the capacitor of the RLC circuit. For simplicity the following terms are defined

$$
\epsilon=\sqrt{\frac{L}{C}}, \quad \alpha=\sigma-\frac{1}{R}, \quad \beta=\frac{3 k_{l}}{\alpha}
$$

where $\sigma, k$ are positive constants. With the natural frequency of oscillation $\omega=\sqrt{L C}$. For the synchronization analysis the equations (8) and (9) need to be transformed into polar coordinates (Johnson et al. (2014a)). This transformation gives an explicit expression of the phase angle of the converter $\theta$ and amplitude $r$ related to the voltage amplitude $v_{c}$. The equations in polar coordinates are,

$$
\frac{d r_{i}}{d t}=\frac{1}{C}\left(\alpha h(r \cos (\omega t-\theta))+k_{l} u(t)\right) \cos (\omega t-\theta)
$$

$$
\frac{d \theta_{i}}{d t}=\omega-\left(\frac{\alpha}{r C} h(r \cos (\omega t-\theta))+\frac{k_{l} u(t)}{r C}\right) \sin (\omega t-\theta)
$$

Where $h(y)$ is the function $h(y)=y-\frac{\beta}{3} y^{3}$.

Notice that equations (10) and (11) depend on the term $k u(t)$ which is the input current (sinusoidal) for the RLC circuit. Equation (11) also depend on the term $\alpha$. As proposed by Johnson et al. (2014b), to ensure a stable limit cycle in the system, $\alpha$ has to be positive and small. This implies that a simplified form for equation (11) can take the form of the equivalent Kuramoto model.

\subsection{Kuramoto equivalent}

From equation (11), if $\alpha$ can be considered as a small, equation (11) becomes,

$$
\frac{d \theta_{i}}{d t}=\omega-\left(\frac{k_{l} u(t)}{r C}\right) \sin (\omega t-\theta)
$$

Such equivalent reduced nonlinear model has the same structure as the Kuramoto model. Being $a_{i j}$ equal to,

$$
\frac{k_{l} u(t)}{r C}=a_{i j}
$$

The comparison of the two equivalent Kuramoto models for different control techniques represents a simplified task compared to the work presented by Johnson et al. (2017), in which the use of the measured current and the protector matrix (Johnson et al. (2014b)), the synchronization error is computed.

\subsection{Discussion}

To the authors understanding, the methods used nowadays for the analysis of synchronization characteristics in the SRFC does not include the complete PLL dynamics for the analysis. An explicit form for the phase angle is needed for the modeling of the PLL dynamics. Such mathematical expression could be used to understand the problematic of the synchrony of converters under adverse circumstances like

- The converters coupled by exchanging power.

- Converters connected to a "weak" utility grid.

- Converters controlling the DC voltage.

This paper presents the fact that there is no formal way to compare converters with different control objectives and structures. It presents the need for a formulation of the equivalent (Kuramoto) model that represent the dynamic of the synchronization.

\section{ACKNOWLEDGEMENTS}

This work was carried out during the tenure of an ERCIM "Alain Bensoussan" Fellowship Programme. 